\title{
A GENERAL PROBLEM OF MINIMIZING AN INTEGRAL WITH DISCONTINUOUS INTEGRAND*
}

BY

\author{
C. F. ROOS†
}

1. Introduction. There are several well known minimum problems which do not readily yield to the classical analysis of the calculus of variations. In some of these, such as the brachistochrone problem of determining the curve of quickest descent from a fixed curve to a fixed point, the integrand of the integral to be minimized is a function of an end value which is variable.f In others, such as that of finding a point $\pi$ and a curve through this point and two fixed points such that the area of the surface of revolution formed by rotating this curve and the ordinate of $\pi$ about the $O X$ axis shall have as small a value as possible, the expression to be minimized is the sum of a point function and a definite integral.§ A more general theory which includes this latter problem as a special case has been given by $\mathrm{E}$. H. Clarke.\| In order to develop a general dynamical theory of depreciation it is desirable to consider a very general problem which somewhat resembles each of the above types, but which does not yield to the analysis of either. I

The depreciation problem as well as the others referred to above can all be considered as special cases of a very general problem with discontinuous integrand, which also includes the most general problems of both the Lagrange and Mayer type with general boundary conditions.** In this paper it is proposed to develop a theory for minimizing an integral whose integrand is a discontinuous function sufficiently general in nature to include all of the the above mentioned types of problems and many others. $\dagger \dagger$

* Presented to the Society, April 16, 1927; received by the editors in December, 1927.

$\dagger$ National Research Fellow.

$\ddagger$ Bliss, Calculus of Variations, pp. 78-79.

$\$$ Mary E. Sinclair, Concerning a compound discontinuous solution in the problem of the surface of revolution of minimum area, Annals of Mathematics, (2), vol. 10, p. 55.

$\|$ E. H. Clarke, On the minimum of the sum of a definite integral and a funstion of a point, Doctoral Dissertation, University of Chicago.

I C. F. Roos, A mathematical theory of depreciation and replacement, American Journal of Mathematics, January, 1928. See also Roos, The problem of depreciation in the calculus of variations, Bulletin of the American Mathematical Society, March-April, 1928.

** O. Bolza, Über den "Anormalen Fall" beim Lagrangeschen und Mayerschen Problem mit gemischten Bedingungen und variablen Endpunkten, Mathematische Annalen, vol. 74 (1913), pp. 430446.

t† For the first discussion of a problem with discontinuous integrand see Mason and Bliss, A problem of the calculus of variations in which the integrand is discontinuous, these Transactions, vol. 7 (1906), pp. 325-336. 
The expression to be minimized is the sum of two integrals taken from $x_{1}$ to a variable limit $\omega$, and from $\omega$ to $x_{2}$, respectively. It may also be regarded as a single integral with an integrand which is discontinuous at the point $\omega, y(\omega)$ of the minimizing curve. The integrand in addition to containing the usual arguments $x, y$ and $d y / d x$ contains also the end values $x_{1}, y\left(x_{1}\right), x_{2}$, $y\left(x_{2}\right)$ and the corner values $\omega, y(\omega)$.

In the work which follows we will find it convenient to use the notation of the tensor calculus to indicate summations. It will be understood, therefore, that when a subscript occurs twice in a term that term is to be summed for all values of the subscript.

2. Statement of the problem. It is desired to find among the values $\omega$ and $\operatorname{arcs} y_{i}=y_{i}(x)\left(i=1, \cdots, n ; x_{1} \leqq x \leqq x_{2}\right)$, satisfying $m$ differential equations,

$$
\phi_{\alpha}\left(x, y, y^{\prime}\right)=0 \quad(\alpha=1, \cdots, m)
$$

in $x$ and $y$, and having end points satisfying end equations

$$
\theta_{\mu}\left(\rho_{1}, \gamma_{1}, \rho_{2}, \gamma_{2}\right)=0 \quad(\mu=1, \cdots, \rho \leqq 2 n+2),
$$

one which minimizes an expression of the form

$$
\begin{aligned}
I= & \int_{x_{1}}^{\omega} g\left(x, y, y^{\prime}, \rho_{1}, \gamma_{1}, \rho_{2}, \gamma_{2}, \omega, y(\omega)\right) d x \\
& +\int_{\omega}^{x_{2}} h\left(x, y, y^{\prime}, \rho_{1}, \gamma_{1}, \rho_{2}, \gamma_{2}, \omega, y(\omega)\right) d x,
\end{aligned}
$$

where for convenience in notation the set $\left(y_{1}, \cdots, y_{n}, y_{1}^{\prime}, \cdots, y_{n}^{\prime}\right)$ has been represented by $\left(y, y^{\prime}\right) ; \gamma_{1}$ and $\gamma_{2}$ are $n$-partite numbers which for a particular curve $y_{i}(x)$ stand respectively for the sets $y_{1}\left(x_{1}\right), \cdots, y_{n}\left(x_{1}\right)$, and $y_{1}\left(x_{2}\right)$, $\cdots, y_{n}\left(x_{2}\right)$, and, finally, primes denote derivatives with respect to $x$.

3. Admissible arcs and variations. In the analysis which follows we will need the following further hypotheses:*

(a) the functions $y_{i}(x)$ defining the minimizing arc $E_{12}$ are continuous on the interval $x_{1}, x_{2}$, and this interval can be subdivided into a finite number of parts on each of which the functions have continuous derivatives;

(b) in a neighborhood $R$ of the values $x, y, y^{\prime}, \omega, y(\omega), x_{1}, y\left(x_{1}\right), x_{2}, y\left(x_{2}\right)$ on the minimizing arc the functions $g, h$ and $\phi_{\alpha}$ have continuous derivatives up to and including those of the second order;

* Bliss, Lectures on the Calculus of Variations, University of Chicago, summer quarter, 1925, mimeographed by O. E. Brown, Northwestern University, Evanston, Ill.; this will be cited as Bliss, Lectures. 
(c) at every element $\left(x, y, y^{\prime}\right)$ on $E_{12}$ the $(m \times n)$-dimensional matrix $\partial \phi_{\alpha} / \partial y_{i}^{\prime}$ has rank $m$, and

(d) the functions $\theta_{\mu}$ have continuous derivatives up to and including those of the second order near the end values $\left(x_{1}, y\left(x_{1}\right), x_{2}, y\left(x_{2}\right)\right)$ of the minimizing arc, and at these end values the $p \times(2 n+2)$-dimensional matrix

$$
\left\|\partial \theta_{\mu} / \partial x_{1} \quad \partial \theta_{\mu} / \partial y\left(x_{1}\right) \quad \partial \theta_{\mu} / \partial x_{2} \quad \partial \theta_{\mu} / \partial y\left(x_{2}\right)\right\|
$$

has rank $p$.

An $\operatorname{arc} y_{i}=y_{i}(x), i=1, \cdots, n ; x_{1} \leqq x \leqq x_{2}$, will be called an admissible arc if it is given by functions $y_{i}(x)$, defined on an interval $\left(x_{1}, x_{2}\right)$, and by a number $\omega_{0}$ such that $x_{1}<\omega_{0}<x_{2}$ and such that the $y_{i}^{\prime}(x)$ are continuous on the intervals $\left(x_{1}, \omega_{0}\right)$ and $\left(\omega_{0}, x_{2}\right)$.

Let us suppose that we have a one-parameter family of admissible arcs given by functions

$$
y_{i}(x, b), \quad x_{1}(b), \quad x_{2}(b) \quad \text { and } \quad \omega(b)
$$

defined on an interval $\left(b_{1}, b_{2}\right)$ such that $y_{i}\left(x, b_{0}\right)=y_{i}(x), x_{1}\left(b_{0}\right)=x_{1}, x_{2}\left(b_{0}\right)=x_{2}$ and $\omega\left(b_{0}\right)=\omega_{0}$, and such that the functions $y_{i}(x, b)$ have continuous firstorder derivatives with respect to $x$ on the intervals $\left[x_{1}(b), \omega(b)\right]$ and $[\omega(b)$, $\left.x_{2}(b)\right]$. We define the functions $\eta_{i}(x)=\partial y_{i}\left(x, b_{0}\right) / \partial b, \xi_{1}=x_{1}^{\prime}\left(b_{0}\right), \xi_{2}=x_{2}^{\prime}\left(b_{0}\right)$, $\xi_{3}=\omega^{\prime}\left(b_{0}\right)$ as the variations of the family along $E_{12}$.

Let us denote by $\Omega_{i}(x)$ the variation $\eta_{i}(x)$ of $y_{i}(x, b)$ for the interval $x_{1} \leqq x \leqq \omega$ and by $\pi_{i}(x)$ the corresponding variation of $y_{i}(x, b)$ for the interval $\omega \leqq x \leqq x_{2}$, and by $\left(u, u^{\prime}\right)$ the set $\left(y, y^{\prime}\right)$ for $x_{1} \leqq x \leqq \omega$ and by $\left(v, v^{\prime}\right)$ the corresponding set $\left(y, y^{\prime}\right)$ for $\omega \leqq x \leqq x_{2}$. We do not assume that $\Omega_{i}(\omega)=\pi_{i}(\omega)$.

The equations of variation for the functions $\phi_{\alpha}$ are defined by

$$
\psi_{\alpha}=\left(\partial \phi_{\alpha} / \partial y_{i}\right) \eta_{i}+\left(\partial \phi_{\alpha} / \partial y_{i}^{\prime}\right) \eta_{i}^{\prime}=0 \quad(\alpha=1, \cdots, m)
$$

where it is understood that $\eta_{i}=\Omega_{i}$ for $x_{1} \leqq x \leqq \omega$ and $\eta_{i}=\pi_{i}$ for $\omega \leqq x \leqq x_{2}$, and the coefficients $\partial \phi_{\alpha} / \partial y_{i}, \partial \phi_{\alpha} / \partial y_{i}^{\prime}$ have as arguments the functions $y_{i}(x)$ defining the minimizing arc $E_{12}$.

The equations of variation for the functions $\theta_{\mu}$ are

$$
\begin{aligned}
{\left[\partial \theta_{\mu} / \partial x_{1}\right.} & \left.+\left(\partial \theta_{\mu} / \partial y_{i}\left(x_{1}\right)\right) y_{i}^{\prime}\left(x_{1}\right)\right] \xi_{1}+\left[\partial \theta_{\mu} / \partial y_{i}\left(x_{1}\right)\right] \eta_{i}\left(x_{1 j}\right. \\
& +\left[\partial \theta_{\mu} / \partial x_{2}+\left(\partial \theta_{\mu} / \partial y_{i}\left(x_{2}\right)\right) y_{i}^{\prime}\left(x_{2}\right)\right] \xi_{2}+\left[\partial \theta_{\mu} / \partial y_{i}\left(x_{2}\right)\right] \eta_{i}\left(x_{2}\right)=0 .
\end{aligned}
$$

A set of functions $\eta_{i}(x)$ with the continuity properties described in (a) except at $x=\omega_{0}$, and satisfying the above equations of variation, (5) and (6), we define as an admissible set of variations. 
TheOREM 1. For every set of admissible variations $\eta_{i}(x)$ along the arc $E_{12}$ there exists a one-parameter family, (4), of admissible arcs containing $E_{12}$ for the value $b=0$ and having the functions $\eta_{i}(x)$ as its variations along $E_{12}$. For this family the functions $y_{i}(x, b)$ are continuous and have continuous derivatives with respect to $b$ for all values, $(x, b)$, near those defining $E_{12}$, and the derivatives $y_{i x}(x, b)$ have the same property except at the values of $x$ on the curve defined by $x=\omega(b), y_{i}=y_{i}(\omega(b), b)$ and, possibly, at the values of $x$ defining other corners of $E_{12}$.

This theorem can be proved by slightly modifying a proof given by Bliss.* Following his example we enlarge the system $\phi_{\alpha}=0$ to have the form

$$
\phi_{1}=0, \cdots, \phi_{m}=0, \phi_{m+1}=z_{m+1}, \cdots, \phi_{n}=z_{n}
$$

where $z_{m+1}, \cdots, z_{n}$ are new variables, and $\phi_{m+1}, \cdots, \phi_{n}$ are new functions of $\left(x, y, y^{\prime}\right)$ such that the functional determinant $\left|\partial \phi_{i} / \partial y_{k}^{\prime}\right|$ is different from zero along $E_{12} . \dagger$ By means of the last $n-m$ of these equations the functions $y_{i}(x)$ belonging to $E_{12}$ define a set of functions $z_{r}(x) \quad(r=m+1$, $\cdots, n)$.

We have a corresponding system of equations of variation,

$$
\psi_{1}=0, \cdots, \psi_{m}=0, \quad \psi_{m+1}=\zeta_{m+1}, \cdots, \psi_{n}=\zeta_{n} \text {, }
$$

along $E_{12}$. The last $n-m$ of these define a set $\zeta_{r}(x)$ corresponding to every set of admissible variations $\eta_{i}(x)$.

Since $\left|\partial \phi_{i} / \partial y_{k}^{\prime}\right| \neq 0$ along $E_{12}$, the existence theorems for differential equations tell us that the system $\ddagger$

$$
\phi_{\alpha}=0, \quad \phi_{r}=z_{r}(x)+b \zeta_{r}(x)
$$

determines uniquely a one-parameter family of solutions,

$$
y_{i}=U_{i}(x, b) \quad\left(x_{1}(b) \leqq x \leqq \omega(b)\right),
$$

with initial conditions

$$
y_{i}\left(x_{1}\right)+b \eta_{i}\left(x_{1}\right)=U_{i}\left(x_{1}, b\right),
$$

* Bliss, Lectures, pp. 4-6.

$\dagger$ For a proof of the possibility of this adjunction see Bliss, The problem of Mayer with variable end points, these Transactions, vol. 19 (1918), p. 312.

$\ddagger$ Bolza, Vorlesungen über Variationsrechnung, pp. $168 \mathrm{ff}$.

Bliss, Annals of Mathematics, (2), vol. 6 (1904), p. 49.

Bliss, Bulletin of the American Mathematical Society, vol. 25 (1918), p. 15. 
where $x=\omega(b)$ is the value of $x$ along a curve of corners $x=\omega(b), u_{i}=v_{i}$ $=y_{i}(\omega(b), b)$ with $\omega(b)>x_{1}(b)$ and $\omega\left(b_{0}\right)=\omega_{0}$ but $\omega(b)$ otherwise arbitrary.

For the interval $\left(\omega(b) \leqq x \leqq x_{2}(b)\right)$ there is a unique one-parameter family of solutions $y_{i}=V_{i}(x, b)$ having initial conditions at $x=\omega$,

$$
V_{i}(\omega(b), b)=U_{i}(\omega(b), b) .
$$

This family consisting of $U_{i}(x, b)$ on $\left(x_{1} \leqq x \leqq \omega\right)$ and $V_{i}(x, b)$ on $(\omega \leqq x$ $\leqq x_{2}$ ) contains $E_{12}$ for $b=0$ and has variations which satisfy the equations (8) with the functions $\zeta_{r}(x)$. The variations of the family are, therefore, identical with the functions $\eta_{i}(x)$ originally prescribed, since when the $\zeta_{r}$ are given, there is only one set of solutions of equations (8) with given initial values $\eta_{i}\left(x_{1}\right)$ at $x=x_{1}$.

Corollary. For a matrix

$$
\left\|\begin{array}{ccc}
\xi_{1}^{(1)} & \cdots & \left.\xi_{1}+1\right) \\
\xi_{2}^{(1)} & \cdots & \xi_{2}^{(p+1)} \\
\eta_{1}^{(1)} & \cdots & \eta_{1}^{(p+1)} \\
\cdots & \cdots & \cdot \cdot \cdot \cdot \\
\eta_{n}^{(1)} & \cdots & \eta_{n}^{(p+1)}
\end{array}\right\|,
$$

whose columns are sets of admissible variations, there exists a $(p+1)$-parameter family, $y_{i}=y_{i}\left(x, b_{1}, \cdots, b_{p+1}\right), x_{1}\left(b_{1}, \cdots, b_{p+1}\right) \leqq x \leqq x_{2}\left(b_{1}, \cdots, b_{p+1}\right)$, containing $E_{12}$ for $\left(b_{1}, \cdots, b_{p+1}\right)=(0, \cdots, 0)$ and having the sets $\xi_{1}{ }^{(0)}$, $\xi_{2}{ }^{(s)}$ and $\eta_{i}^{\left({ }^{(}\right)}(x)$ as its variations along $E_{12}$ with respect to the parameter $b_{.}$. The continuity properties are similar to those described in the above theorem.

This corollary is proved with the equations $\phi_{\alpha}=0, \cdots, \phi_{r}=z_{r}+b_{1} \zeta_{r}^{(1)}$ $+\cdots+b_{p+1} \zeta_{r}^{(p+1)}$ replacing equations (9).

4. First variation of the integral $I$. If the one-parameter family of admissible arcs (4) be substitued in (3), we obtain for the first variation of the resulting expression for $b=b_{0}=0$

$$
\begin{aligned}
I_{1}(\xi, \eta)= & \int_{x_{1}}^{\omega_{0}}\left[\left(\partial g / \partial u_{i}\right) \Omega_{i}+\left(\partial g / \partial u_{i}^{\prime}\right) \Omega_{i}^{\prime}\right] d x+\int_{\omega_{0}}^{x_{2}}\left[\left(\partial h / \partial v_{i}\right) \pi_{i}\right. \\
& \left.+\left(\partial h / \partial v_{i}^{\prime}\right) \pi_{i}^{\prime}\right] d x+S_{1 i}(g, h) \Omega_{i}\left(x_{1}\right)+S_{2 i}(g, h) \pi_{i}\left(x_{2}\right)+L_{1}(g, h) \xi_{1} \\
& +L_{2}(g, h) \xi_{2}+M(g, h) \omega^{\prime}(0)+N_{i}(g, h) \frac{d y_{i}(\omega(0), 0)}{d b}
\end{aligned}
$$

where as notation 


$$
\begin{aligned}
& S_{\sigma i}(g, h)=\int_{x_{1}}^{\omega_{0}}\left(\partial g / \partial y_{i}\left(x_{\sigma}\right)\right) d x+\int_{\omega_{0}}^{x_{2}}\left(\partial h / \partial y_{i}\left(x_{\sigma}\right)\right) d x \quad(\sigma=1,2) ; \\
& L_{\sigma}(g, h)=(\sigma-2) g\left(x_{1}\right)+(\sigma-1) h\left(x_{2}\right)+\int_{x_{1}}^{\omega_{0}}\left[\partial g / \partial x_{\sigma}+\left(\partial g / \partial y_{i}\left(x_{\sigma}\right)\right) y_{i}^{\prime}\left(x_{\sigma}\right)\right] d x \\
& +\int_{\omega_{0}}^{x_{2}}\left[\partial h / \partial x_{\sigma}+\left(\partial h / \partial y_{i}\left(x_{\sigma}\right)\right) y_{i}^{\prime}\left(x_{\sigma}\right)\right] d x ;
\end{aligned}
$$$$
M(g, h)=g\left(\omega_{0}\right)-h\left(\omega_{0}\right)+\int_{x_{1}}^{\omega_{0}}(\partial g / \partial \omega) d x+\int_{\omega_{0}}^{x_{2}}(\partial h / \partial \omega) d x ;
$$$$
N_{i}(g, h)=\int_{x_{1}}^{\omega_{0}}\left[\partial g / \partial u_{i}(\omega)\right] d x+\int_{\omega_{0}}^{x_{2}}\left[\partial h / \partial v_{i}(\omega)\right] d x ;
$$

$g\left(x_{1}\right)$ and $g\left(\omega_{0}\right)$ are the values of $g$ at the points (1) and $\left(\omega_{0}\right)$ on $E_{12} ; h\left(\omega_{0}\right)$ and $h\left(x_{2}\right)$ are the values of $h$ at the points $\left(\omega_{0}\right)$ and (2) on $E_{12} ; i$ is an umbral index with range $1, \cdots, n$ when it occurs twice as a subscript, and $\sigma$ is not umbral in $L_{\boldsymbol{c}}$.

Let us multiply each of the equations (8) by a function $\lambda(x)$; form the $\operatorname{sum} \lambda_{\alpha} \psi_{\alpha}+\lambda_{r} \psi_{r}-\lambda_{r} \zeta_{r}$ where $\alpha$ and $r$ are umbral indices with ranges $1, \cdots, m$ and $m+1, \cdots, n$, respectively, and then integrate this sum from $x_{1}$ to $x_{2}$. We obtain

$$
\begin{aligned}
\int_{x_{1}}^{\omega_{0}}\left[\left(\partial R / \partial u_{i}\right) \Omega_{i}+\left(\partial R / \partial u_{i}^{\prime}\right) \Omega_{i}^{\prime}\right] d x \\
\quad+\int_{\omega_{0}}^{x_{2}}\left[\left(\partial T / \partial v_{i}\right) \pi_{i}+\left(\partial T / \partial v_{i}^{\prime}\right) \pi_{i}^{\prime}\right] d x-\int_{x_{1}}^{x_{2}} \lambda_{r} \zeta d x=0,
\end{aligned}
$$

where, by definition, $R=\lambda_{i} \phi_{i}, i=1, \cdots, n$, on $x_{1} \leqq x \leqq \omega$, and $T=\lambda_{i} \phi_{i}$ on $\omega \leqq x \leqq x_{2}$. It seems desirable to split the integral into two parts in this manner because this was done with the original integral $I$.

By (6) the equations of variation on $E_{12}$ for the functions $\theta_{\mu}$ are

$$
\begin{aligned}
K_{\mu}(\xi, \eta)= & {\left[\left(\partial \theta_{\mu} / \partial x_{1}\right)+\left(\partial \theta_{\mu} / \partial y_{i}\left(x_{1}\right)\right) y_{i}^{\prime}\left(x_{1}\right)\right] \xi_{1}+\left[\partial \theta_{\mu} / \partial y_{i}\left(x_{1}\right)\right] \Omega_{i}\left(x_{1}\right) } \\
& +\left[\partial \theta_{\mu} / \partial x_{2}+\left(\partial \theta_{\mu} / \partial y_{i}\left(x_{2}\right)\right) y_{i}^{\prime}\left(x_{2}\right)\right] \xi_{2}+\left[\partial \theta_{\mu} / \partial y_{i}\left(x_{2}\right)\right] \pi_{i}\left(x_{2}\right)=0 .
\end{aligned}
$$

If a $(p+1)$-parameter family of admissible arcs $y_{i}=y_{i}\left(x, b_{1}, \cdots, b_{p+1}\right)$, $x_{1}\left(b_{1}, \cdots, b_{p+1}\right), x_{2}\left(b_{1}, \cdots, b_{p+1}\right), \omega\left(b_{1}, \cdots, b_{p+1}\right)$, containing $E_{12}$ for $\left(b_{1}, \cdots, b_{p+1}\right)=(0, \cdots, 0)$, be substituted in $I$ and the functions $\theta_{\mu}$, these become functions of $b_{1}, \cdots, b_{p+1}$. If $I_{0}$ is the desired minimum yielded by $E_{12}$, then the first members of the equations

$$
\begin{aligned}
I\left(b_{1}, \cdots, b_{p+1}\right) & =I_{0}+q, \\
\theta_{\mu}\left(b_{1}, \cdots, b_{p+1}\right) & =0,
\end{aligned}
$$


where $q$ is a positive number, must have their functional determinant equal to zero for $\left(b_{1}, \cdots, b_{p+1}\right)=(0, \cdots, 0)$, since, otherwise, these equations would have solutions near this set of values for both positive and negative values of $q$. This functional determinant of rank $r<p+1$ is

$$
\left|\begin{array}{c}
I_{1}\left(\xi^{(1)}, \eta^{(1)} \cdots \cdots I_{1}\left(\xi^{(p+1)}, \eta^{(p+1)}\right)\right. \\
K_{1}\left(\xi^{(1)}, \eta^{(1)}\right) \cdots \cdot K_{1}\left(\xi^{(p+1)}, \eta^{(p+1)}\right) \\
\cdots \cdots \cdots \cdots \\
K_{p}\left(\xi^{(1)}, \eta^{(1)}\right) \cdots \cdot K_{p}\left(\xi^{(p+1)}, \eta^{(p+1)}\right)
\end{array}\right|,
$$

and from its vanishing we argue that there exists a set of constants $d_{1}, \cdots$, $d_{p}, \lambda_{0}$, not all zero, such that the equation

$$
\lambda_{0} I_{1}(\xi, \eta)+d_{\mu} K_{\mu}(\xi, \eta)=0 \quad(\mu \text { umbral with range } 1, \cdots, p)
$$

holds for every set of admissible variations $\xi_{1}, \xi_{2}, \omega^{\prime}(0), \eta(x)$, since, otherwise, one of the columns of this determinant could be replaced by another making it of rank $r+1$. $^{*}$

As notation let $\nu$ be an umbral index with range 1,2. Let us define two new functions $G$ and $B$ by the respective equations

$$
\begin{aligned}
G\left(x, y, y^{\prime}, x_{1}, \cdots, y\left(x_{2}\right), \lambda\right) & =\lambda_{0} g+\lambda_{i} \phi_{i}, \\
H\left(x, y, y^{\prime}, x_{1}, \cdots, y\left(x_{2}\right), \lambda\right) & =\lambda_{0} h+\lambda_{i} \phi_{i} .
\end{aligned}
$$

Since the variations $(\xi, \eta)$ satisfy (13), the value of (16) is not altered if we add (13) to it. By the help of (12) we can, therefore, write

$$
\begin{aligned}
& \int_{x_{1}}^{\omega_{0}}\left[\left(\partial G / \partial u_{i}\right) \Omega_{i}+\left(\partial G / \partial u_{i}^{\prime}\right) \Omega_{i}^{\prime}-\lambda_{r} \zeta_{r}\right] d x \\
+ & \int_{\omega_{0}}^{x_{2}}\left[\left(\partial H / \partial v_{i}\right) \pi_{i}+\left(\partial H / \partial v_{i}^{\prime}\right) \pi_{i}^{\prime}-\lambda_{r} \zeta_{r}\right] d x+\left[S_{v_{i}}(g, h)\right. \\
+ & \left.d_{\mu}\left(\partial \theta_{\mu} / \partial y_{i}\left(x_{\nu}\right)\right)\right] \eta_{i}\left(x_{\nu}\right)+\left[L_{\nu}(g, h)+d_{\mu}\left(\partial \theta_{\mu} / \partial x_{\nu}\right)\right] \xi_{\nu} \\
+ & {\left[\left(\partial \theta_{\mu} / \partial y_{i}\left(x_{1}\right)\right) y_{i}^{\prime}\left(x_{1}\right)\right] \xi_{1}+\left[\left(\partial \theta_{\mu} / \partial y_{i}\left(x_{2}\right)\right) y_{i}^{\prime}\left(x_{2}\right)\right] \xi_{2}+M(g, h) \omega^{\prime}(0) } \\
+ & N_{i}(g, h) d y_{i}(\omega(0), 0) / d b=0 .
\end{aligned}
$$

So far the functions $\lambda_{i}(x)$ have been entirely arbitrary.

5. First necessary conditions. We now proceed to determine the $\lambda_{i}(x)$ so that the equations

* Bliss, Lectures, p. 20. 


$$
\begin{array}{ll}
\partial G / \partial u_{i}^{\prime}=\int_{x_{1}}^{x}\left(\partial G / \partial u_{i}\right) d x+C_{i 1} & \left(x_{1} \leqq x \leqq \omega\right), \\
\partial H / \partial v_{i}^{\prime}=\int_{x}^{x_{2}}\left(\partial H / \partial v_{i}\right) d x+C_{i 2} & \left(\omega \leqq x \leqq x_{2}\right)
\end{array}
$$

are satisfied for an arbitrarily selected set of constants $C_{i 1}, C_{i 2}$. This is possible because of the assumption that $\left|\partial \phi_{i} / \partial y_{k}^{\prime}\right|$ is different from zero along $E_{12}$. For the details of the proof of the possibility of this choice of the $\lambda_{i}$ we refer the reader to the mimeographed lecture notes of G. A. Bliss, ${ }^{*}$ since the proof which Bliss gives there requires only slight alteration. For this choice of the functions $\lambda_{i}(x)$ equation (17) becomes

$$
\begin{aligned}
& -\int_{x_{1}}^{\omega_{0}} \lambda_{r} \zeta_{r} d x-\int_{\omega_{0}}^{x_{2}} \lambda_{r} \zeta_{r} d x+\left[P_{i}\left(x_{v}\right)+d_{\mu}\left(\partial \theta_{\mu} / \partial y_{i}\left(x_{v}\right)\right)\right] \eta_{i}\left(x_{v}\right)+\left[L\left(x_{v}\right)\right. \\
& \left.+d_{\mu}\left(\partial \theta_{\mu} / \partial x_{v}\right)\right] \xi_{\nu}+\left[d_{\mu}\left(\partial \theta_{\mu} / \partial y_{i}\left(x_{1}\right)\right) y_{i}^{\prime}\left(x_{1}\right)\right] \xi_{1}+\left[d_{\mu}\left(\partial \theta_{\mu} / \partial y_{i}\left(x_{2}\right)\right) y_{i}^{\prime}\left(x_{2}\right)\right] \xi_{2} \\
& \quad+M(g, h) \omega^{\prime}(0)+\left[\partial G / \partial u^{\prime}\left(\omega_{0}\right)\right] \Omega_{i}\left(\omega_{0}\right)-\left[\partial H / \partial v_{i}^{\prime}\left(\omega_{0}\right)\right] \pi_{i}\left(\omega_{0}\right) \\
& \quad+\left[N_{i}(g, h)\right] d y_{i}\left(\omega_{0}, 0\right) / d b=0
\end{aligned}
$$

where

$P_{i}\left(x_{\nu}\right)=(\nu-2)\left(\partial G / \partial u_{i}^{\prime}\left(x_{1}\right)+(\nu-1)\left(\partial H / \partial v_{i}^{\prime}\left(x_{2}\right)\right)+S_{\nu i}(g, h) \quad(\nu=1,2)\right.$,

and $L\left(x_{v}\right)=L_{\nu}(g, h) ; \partial G / \partial u_{i}^{\prime}\left(x_{1}\right)$ and $\partial G / \partial u_{i}^{\prime}\left(\omega_{0}\right)$ denote derivatives with respect to $u_{i}^{\prime}$ at the point $(1)$ and $\left(\omega_{0}\right)$, respectively, on $E_{12}$, and $\partial H / \partial v_{i}^{\prime}\left(\omega_{0}\right)$ and $\partial H / \partial v_{i}^{\prime}\left(x_{2}\right)$ denote derivatives of $H$ with respect to $v_{i}^{\prime}$ at the points $\left(\omega_{0}\right)$ and (2), respectively, on $E_{12}$, and where we have chosen the constants $C_{i 1}$ and $C_{i 2}$ so that equations (18) and (19) are satisfied, i.e., $C_{i 1}=\partial G / \partial u_{i}^{\prime}\left(x_{1}\right)$ and $C_{i z}=\partial H / \partial v_{i}^{\prime}\left(x_{2}\right)$.

Since the extremal $E_{12}$ is continuous at $x=\omega_{0}$ the equations $y_{i}(\omega(b), b)$ $=u_{i}(\omega(b), b)=v_{i}(\omega(b), b)$ must hold, and, hence, by a differentiation

$$
d y_{i}\left(\omega_{0}, 0\right) / d b=u_{i}^{\prime}\left(\omega_{0}\right) \omega^{\prime}(0)+\Omega_{i}\left(\omega_{0}\right)=v_{i}^{\prime}\left(\omega_{0}\right) \omega^{\prime}(0)+\pi_{i}\left(\omega_{0}\right) .
$$

If we substitute the values of $\Omega_{i}\left(\omega_{0}\right)$ and $\pi_{i}\left(\omega_{0}\right)$ defined by (21) in (20), we obtain as a necessary condition for a minimum of the integral $I$

$$
\begin{aligned}
& -\int_{x_{1}}^{\omega_{0}} \lambda_{r} \zeta_{r} d x-\int_{\omega_{0}}^{x_{2}} \lambda_{r} \zeta_{r} d x+\left[P_{i}\left(x_{v}\right)+d_{\mu}\left(\partial \theta_{\mu} / \partial y_{i}\left(x_{v}\right)\right)\right] \eta_{i}\left(x_{v}\right)+\left[L\left(x_{v}\right)\right. \\
& \left.\quad+d_{\mu}\left(\partial \theta_{\mu} / \partial x_{\nu}\right)\right] \xi_{\nu}+\left[d_{\mu}\left(\partial \theta_{\mu} / \partial y_{i}\left(x_{1}\right)\right) y_{i}^{\prime}\left(x_{1}\right)\right] \xi_{1}+\left[d_{\mu}\left(\partial \theta_{\mu} / \partial y_{i}\left(x_{2}\right)\right) y_{i}^{\prime}\left(x_{2}\right)\right] \xi_{2} \\
& \quad+\left[-u^{\prime}\left(\omega_{0}\right) \partial G / \partial u_{i}^{\prime}\left(\omega_{0}\right)+v_{i}^{\prime}\left(\omega_{0}\right) \partial H / \partial v_{i}^{\prime}\left(\omega_{0}\right)+M(g, h)\right] \omega^{\prime}(0) \\
& \quad+\left[\partial G / \partial u_{i}^{\prime}\left(\omega_{0}\right)-\partial H / \partial v_{i}^{\prime}\left(\omega_{0}\right)+N_{i}(g, h)\right] d y_{i}\left(\omega_{0}, 0\right) / d b=0 .
\end{aligned}
$$

\footnotetext{
* Bliss, Lectures, p. 7.
} 
Since $\omega^{\prime}(0)$ and $\Omega_{i}\left(\omega_{0}\right)$ are arbitrary, equations (21) show us that $d y_{i}\left(\omega_{0}\right.$, $0) / d b$ is arbitrary. We can, therefore, say that the expression (22) must vanish for every choice of $\eta_{i}\left(x_{v}\right), \xi_{v}, \omega^{\prime}(0), d y_{i}\left(\omega_{0}, 0\right) / d b$ and $\zeta_{r}, r=m+1, \cdots, n$. It follows then that all of the coefficients of these quantities must vanish and, hence, in particular, that $\lambda_{m+1} \equiv \cdots \equiv \lambda_{n} \equiv 0$. It follows further with the help of (12) that

$$
\begin{aligned}
- & u_{i}^{\prime}\left(\omega_{0}\right)\left(\partial G / \partial u_{i}^{\prime}\left(\omega_{0}\right)+v_{i}^{\prime}\left(\omega_{0}\right)\left(\partial H / \partial v_{i}^{\prime}\left(\omega_{0}\right)\right)+G\left(\omega_{0}\right)-H\left(\omega_{0}\right)\right. \\
+ & \int_{x_{:}}^{\omega_{0}}(\partial G / \partial \omega) d x+\int_{\omega_{0}}^{x_{2}}(\partial H / \partial \omega) d x=0, \\
\partial G / \partial u_{i}^{\prime}\left(\omega_{0}\right)-\partial H / \partial v_{i}^{\prime}\left(\omega_{0}\right)+\int_{x}^{\omega_{0}}\left(\partial G / \partial u_{i}\left(\omega_{0}\right)\right) d x & \\
& +\int_{\omega_{0}}^{x_{2}}\left(\partial H / \partial v_{i}\left(\omega_{0}\right)\right) d x=0 \quad(i=1, \cdots, n),
\end{aligned}
$$

where as already stated $u_{i}$ refers to the extremal arc $y_{i}$ for the interval $x_{1} \leqq x$ $\leqq \omega_{0}$, and $v_{i}$ refers to the same arc $y_{i}$ but for the interval $\omega_{0} \leqq x \leqq x_{2}$; further that in the matrix

$$
\left\|\begin{array}{llll}
L\left(x_{1}\right) & P_{i}\left(x_{1}\right) & L\left(x_{2}\right) & P_{i}\left(x_{2}\right) \\
\theta_{\mu x_{1}}+\theta_{\mu y_{1} 1} y_{i 1}^{\prime} & \theta_{\mu y_{i 1}} & \theta_{\mu x_{2}}+\theta_{\mu y_{i} 2} y_{i 2}^{\prime} & \theta_{\mu y_{i 2}}
\end{array}\right\|,
$$

where $\theta_{\mu x_{1}}+\theta_{\mu v_{i} \nu} y_{i \nu}^{\prime}$ stands for $\partial \theta_{\mu} / \partial x_{1}+\left(\partial \theta_{\mu} / \partial y_{i}\left(x_{\nu}\right)\right) y_{i}^{\prime}\left(x_{\nu}\right)$, every determinant of order $p+1$ vanishes, and finally that the equations (18) and (19) are satisfied. The condition (24) must hold since the multipliers $1, d_{1}, \cdots, d_{p}$ satisfy all the linear equations whose coefficients are columns of the matrix. The rank of this matrix is unchanged when one column is multiplied by a factor and added to another, so that we can state the following theorem.

THEOREM 2. For every minimizing arc for this general problem there exist sets of constants $C_{i 1}$ and $C_{i 2}, i=1, \cdots, n$, and functions

$$
\begin{aligned}
& G\left(x, y, y^{\prime}, x_{1}, y\left(x_{1}\right), \cdots, y\left(x_{2}\right), \lambda\right)=\lambda_{0} g+\lambda_{\alpha} \phi_{\alpha} \quad\left(x_{0} \leqq x \leqq \omega_{0}\right), \\
& H\left(x, y, y^{\prime}, x_{1}, y\left(x_{1}\right), \cdots, y\left(x_{2}\right), \lambda\right)=\lambda_{0} h+\lambda_{\alpha} \phi_{\alpha} \quad\left(\omega_{0} \leqq x \leqq x_{2}\right),
\end{aligned}
$$

such that the equations

$$
\begin{array}{ll}
\partial G / \partial u_{i}^{\prime}=\int_{x}^{x}\left(\partial G / \partial u_{i}\right) d x+C_{i 1} & \left(x_{1} \leqq x \leqq \omega\right), \\
\partial H / \partial v_{i}^{\prime}=\int_{x}^{x_{2}}\left(\partial H / \partial v_{i}\right) d x+C_{i 2} & \left(\omega \leqq x \leqq x_{2}\right)
\end{array}
$$

are satisfied at every point of $E_{12}, x_{1} \leqq x \leqq x_{2}$. The constant $\lambda_{0}$ and the functions 
$\lambda_{\alpha}(x), \alpha=1, \cdots, m$, are not all identically zero on $E_{12}$, and are continuous except possibly at values of $x$ defining corners of $E_{12}$. At the point $\omega, y_{i}(\omega)$, at which there exists a corner, the equations (23), which are the analogues of the Weierstrass-Erdmann corner conditions, must be satisfied. Furthermore, the end values of $E_{12}$ must be such that all determinants of order $p+1$ of the matrix

vanish.

$$
\left\|\begin{array}{cccc}
L\left(x_{1}\right)-y_{i 1}^{\prime} P_{i}\left(x_{1}\right) & P_{i}\left(x_{1}\right) & L\left(x_{2}\right)-y_{i 2}^{\prime} P_{i}\left(x_{2}\right) & P_{i}\left(x_{2}\right) \\
\theta_{\mu x_{1}} & \theta_{\mu y_{i 1}} & \theta_{\mu x_{2}} & \theta_{\mu y_{i 2}}
\end{array}\right\|
$$

These last conditions are the transversality conditions. It is easily seen that if the functions $g$ and $h$ are independent of the end and corner variables they reduce to the corresponding conditions obtained by Bliss.*

The corner conditions as obtained in this paper differ from the corner conditions usually obtained because of the occurrence of the integrals involving derivatives with respect to corner variables, so that if $g$ and $h$ are independent of these variables, the integral terms drop out. For such problems as Miss Sinclair's and E. H. Clarke's these derivatives reduce to terms simple enough to be integrated by inspection. $\dagger$

It will be interesting to examine a few special cases of the very general problem of this paper. In as much as the paper was suggested by the problem of depreciation it will be well to begin with a short examination of this important special case.

6. Some special cases. In order to determine the best time at which to replace a machine, which is in operation, by another machine whose operating expense is different we must maximize an expression which can be thrown in the general form (3). This problem of replacement in dynamical economics is that of finding among the $\operatorname{arcs} y_{i}=y_{i}(x)$ satisfying a differential equation of demand, $D\left(x, y_{1}, y_{1}^{\prime}, y_{2}, y_{2}^{\prime}\right)=0$, where $x$ is the time, $y_{1}$ is the rate of production at the time $x$, and $y_{2}$ is the price at the time $x$, and end conditions

$$
\theta_{\mu}\left(x_{1}, y_{1}\left(x_{1}\right), y_{2}\left(x_{1}\right), x_{2}, y_{1}\left(x_{2}\right), y_{2}\left(x_{2}\right)\right)=0 \quad(\mu=1, \cdots, p \leqq 6),
$$

a set which maximizes an expression of the form

$$
\begin{aligned}
V= & \int_{x_{1}}^{\omega}\left[y_{1} y_{2}-Q_{1}\left(x, y_{1}, y_{2}, y_{1}^{\prime}, y_{2}^{\prime}\right)\right] e^{\beta\left(x_{1}-x\right)} d x \\
& +\int_{\omega}^{x_{2}}\left[y_{1} y_{2}-Q_{2}\left(x, y_{1}, y_{2}, y_{1}^{\prime}, y_{2}^{\prime}\right)\right] e^{\beta\left(x_{1}-x\right)} d x \\
& +C_{1} e^{\beta\left(x_{1}-\omega\right)}+C_{2} e^{\beta\left(x_{1}-x_{2}\right)},
\end{aligned}
$$

* Bliss, The problem of Mayer with variable end points, loc. cit.

$\dagger$ See fourth and fifth footnotes on page 58 . 
where $x$ is the time; $V$ is the value at $x=x_{1}$ of the composite machine, consisting of a machine operating for the period $x_{1} \leqq x \leqq \omega$, and a replacement machine operating for the period $\omega \leqq x \leqq x_{2} ; y_{1}$ is the number of units of output per unit time; $y_{2}$ is the price of one unit of output at the time $x ; Q_{1}$ is the cost of producing $y_{1}$ units of output for the machine called the operating machine; $Q_{2}$ is the cost of producing $y_{1}$ units of output for the replacement machine, and exp. $\left(\beta\left(x_{1}-x\right)\right)$ etc. are discount factors to allow for interest.

Since $\omega$ and $x_{1}$ are constant so far as integration with respect to $x$ is concerned, we may write this expression in the form

$$
\begin{aligned}
V=\int_{x_{1}}^{\omega}\left[\left(y_{1} y_{2}-Q_{1}\right) e^{\beta\left(x_{1}-x\right)}\right. & \left.+\frac{C_{1} e^{\beta\left(x_{1}-\omega\right)}+C_{2} e^{\beta\left(x_{1}-x_{2}\right)}}{\omega-x_{1}}\right] d x \\
& +\int_{\omega}^{x_{2}}\left(y_{1} y_{2}-Q_{2}\right) e^{\beta\left(x_{1}-x\right)} d x .
\end{aligned}
$$

When $V$ is in this form, the replacement problem is easily seen to be a special case of the general problem of this paper. In as much as I have presented a mathematical theory of depreciation and replacement in another paper, there is no need to carry the problem further here.*

As already mentioned, problems which require that the sum of a point function and a definite integral be minimized are special cases of a general problem discussed by Clarke. $\dagger$ His problem is that of finding among the $\operatorname{arcs} y_{i}=y_{i}(x)$ satisfying end conditions $x_{1}=\alpha_{1}, x_{2}=\alpha_{2}, y_{i}\left(x_{1}\right)=\beta_{i 1}, y_{i}\left(x_{2}\right)=\beta_{i 2}$ $(i=1, \cdots, n)$, a set which minimizes the sum of a definite integral and a function of a point,

$$
J=\phi(\omega, y(\omega))+\int_{x_{1}}^{x_{2}} f\left(x, y, y^{\prime}\right) d x .
$$

Although Clarke does not discuss the Lagrange problem analogous to the case above which he studied, the analysis of my paper is sufficiently general to apply to his problem. We may write $J$ in the form

$$
J=\int_{x_{1}}^{\omega}\left[f\left(x, y, y^{\prime}\right)+\frac{\phi(\omega, y(\omega))}{\omega-x_{1}}\right] d x+\int_{\omega}^{x_{2}} f\left(x, y, y^{\prime}\right) d x,
$$

and again obtain a special case. For Clarke's problem the corner conditions (23) become

\footnotetext{
* Roos, $A$ mathematical theory of depreciation and replacement, loc. cit.

$\dagger$ Clarke, On the minimum of the sum etc., loc. cit.
} 


$$
\begin{aligned}
& \phi_{\omega}+f\left(\omega, y(\omega), u^{\prime}(\omega)\right)- f\left(\omega, y(\omega), v^{\prime}(\omega)\right)-u^{\prime}(\omega) f_{u}^{\prime}\left(\omega, y(\omega), u^{\prime}(\omega)\right) \\
&+ v^{\prime}(\omega) f_{v}^{\prime}\left(\omega, y(\omega), u^{\prime}(\omega)\right)=0, \\
& \phi_{v_{i}(\omega)}+f_{u_{i^{\prime}}}^{\prime}\left(\omega, y(\omega), u^{\prime}(\omega)\right)-f_{v_{i^{\prime}}}\left(\omega, y(\omega), v^{\prime}(\omega)\right)=0 \quad(i=1, \cdots, n),
\end{aligned}
$$

and these are identical with the conditions obtained by Clarke, except for notation.

Let us next consider special cases for which the functions $g$ and $h$ are independent of the corner variables and, furthermore, for which $g \equiv h$, i.e., for which there is no corner at $x=\omega$.

An important example of this type of problem is the following proposed by Bolza.

Minimize the expression

$$
U=\int_{x_{0}}^{x_{1}} H\left(y_{1}, \cdots, y_{n}, y_{1}^{\prime}, \cdots, y_{n}^{\prime}\right) d x+G\left(y_{10}, \cdots, y_{n 0}, y_{11}, \cdots, y_{n 1}\right),
$$

when the admissible curves are defined by functions $y_{i}=y_{i}(x)\left(x_{0} \leqq x \leqq x_{1}\right)$ which satisfy differential equations $\phi_{\alpha}\left(y_{i}, y_{i}^{\prime}\right)=0(\alpha=1, \cdots, m)$, while the end points, $y_{i 0}, y_{i 1}$, satisfy conditions $\theta_{\mu}\left(y_{i 0}, y_{i 1}\right)=0(\mu=1, \cdots, p)$. Bolza admits so-called finite equations $\psi_{k}\left(y_{1}, \cdots, y_{n}\right)=0(k=1, \cdots, r<n-m)$, in addition to the equations $\phi_{\alpha}=0$, but, as Bliss has shown, this gives no additional generalization.* Bolza's problem is the special case for which $\phi_{\alpha}$ and $\theta_{\mu}$ of the general problem do not depend upon $x$ explicitly and for which

$$
g \equiv h \equiv \frac{G\left(y_{10}, \cdots, y_{n 0}, y_{11}, \cdots, y_{n 1}\right)}{x_{1}-x_{0}}+H\left(y_{1}, \cdots, y_{n}, y_{1}^{\prime}, \cdots, y_{n}^{\prime}\right)
$$

The integrand is continuous at $x=\omega$ and the corner conditions vanish identically unless $y_{i}(x)$ has a corner there, in which case they reduce to the Weierstrass-Erdmann corner conditions. $\dagger$

In as much as Bolza has shown that his problem is sufficiently general to include as special cases the most general Lagrange problem with general boundary conditions for $G \equiv 0$, and the most general Mayer problem with general boundary conditions for the case $H \equiv 0, G \equiv y_{10}$, it appears that the problem treated in this memoir is sufficiently general to include a very large class of the problems of the calculus of variations.

7. Further generalizations. In the replacement problem discussed in the last paragraph we supposed that there was only one machine producing

* Bliss, The problem of Mayer with variable end points, loc. cit.

† J. Hadamard, Leçons sur le Calcul des Variations, vol. 1, p. 188. 
the commodity or service under consideration at any time $x$. If we suppose several machines to be simultaneously in operation producing the same article, there are two problems of interest. If all owners agree to cooperate in making the sum of their profits a maximum the problem is a special case of the one considered in this paper. On the other hand if the machines are competing, the problem is one for which it is required to obtain partial maxima of as many integrals as there are competing machines. I have already considered the competition case for the special case in which $g$ and $h$ are independent of variable corner and end values.*

In any actual case we do not have all machines in operation for the same period of time, for new competitors are constantly entering and others are dropping out. Such a problem becomes difficult even for the cooperation case because we must maximize an expression which is the sum of say $n$ integrals whose limits may or may not be the same and whose integrands depend upon different numbers of variables $x$ and $y_{i}$. Each time a newcomer produces, the demand equation contains an additional variable, e.g., this producer's rate of production.

* Roos, Generalized Lagrange problems in the calculis of variations, these Transactions, April, 1928. See also Roos, $A$ mathematical theory of competition, American Journal of Mathematics, vol. 47 (1925), pp. 163-175, and Roos, $A$ dynamical theory of economics, Journal of Political Economy, vol. 35 (1927), pp. 632-656.

University of Chicago, Chicago, Irl. 